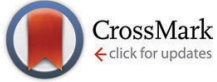

Cite this: Phys. Chem. Chem. Phys., 2016, 18, 4668

Received 13th November 2015 Accepted 7th January 2016

DOI: $10.1039 / c 5 c p 06952 a$

www.rsc.org/pccp

\section{Enhancing graphene capacitance by nitrogen: effects of doping configuration and concentration}

\begin{abstract}
Cheng Zhan, ${ }^{a}$ Yu Zhang, ${ }^{b}$ Peter T. Cummings ${ }^{b}$ and De-en Jiang ${ }^{{ }^{a}}$
Recent experiments have shown that nitrogen doping enhances capacitance in carbon electrode supercapacitors. However, a detailed study of the effect of $\mathrm{N}$-doping on capacitance is still lacking. In this paper, we study the doping concentration and the configuration effect on the electric double-layer (EDL) capacitance, quantum capacitance, and total capacitance. It is found that pyridinic and graphitic nitrogens can increase the total capacitance by increasing quantum capacitance, but pyrrolic configuration limits the total capacitance due to its much lower quantum capacitance than the other two configurations. We also find that, unlike the graphitic and pyridinic nitrogens, the pyrrolic configuration's quantum capacitance does not depend on the nitrogen concentration, which may explain why some capacitance versus voltage measurements of $\mathrm{N}$-doped graphene exhibit a $\mathrm{V}$-shaped curve similar to that of undoped graphene. Our investigation provides a deeper understanding of the capacitance enhancement of the $\mathrm{N}$-doping effect in carbon electrodes and suggests a potentially effective way to optimize the capacitance by controlling the type of $\mathrm{N}$-doping.
\end{abstract}

\section{Introduction}

Electric double-layer capacitors (EDLCs), also called supercapacitors, store electric energy physically at the electrode-electrolyte interface by the formation of the electric double layer under a bias voltage. ${ }^{1}$ The specific capacitance of an EDLC can be much higher than that of a conventional dielectric capacitor. ${ }^{2}$ In recent years, carbon nanomaterials such as carbide-derived carbons and graphene have become popular as supercapacitor electrode materials due to their good conductivity and high specific surface area. ${ }^{2-9}$ Graphene, unlike a traditional metal electrode, has a different capacitive performance due to its quantum capacitance ${ }^{10-12}$ which is caused by its limited density of states (DOS) near the Fermi level.

In graphene supercapacitors, total capacitance $C_{\text {total }}$ reflects the overall effect of quantum capacitance $C_{\mathrm{Q}}$ and the electric double-layer (EDL) capacitance $C_{\mathrm{EDL}}$ and can be estimated by

$$
\frac{1}{C_{\mathrm{total}}}=\frac{1}{C_{\mathrm{Q}}}+\frac{1}{C_{\mathrm{EDL}}}
$$

if one ignores the polarization effect of the electrolyte on the electronic structure of the electrode surface. ${ }^{13}$ Under this assumption, one can treat quantum and EDL capacitances separately to study the total capacitance..$^{8,14,15}$ The contribution

\footnotetext{
${ }^{a}$ Department of Chemistry, University of California, Riverside, CA, 92521, USA. E-mail: de-en.jiang@ucr.edu; Tel: +1-951-827-4430

${ }^{b}$ Department of Chemical and Biomolecular Engineering, Vanderbilt University, Nashville, TN, 37235, USA
}

of the quantum capacitance to the total capacitance is negligible when the electrode is a metal such as Pt which has extremely large DOS near the Fermi level. However, in graphene and graphene-like 2D systems, the quantum capacitance is significant because it is comparable to the EDL capacitance.

A consequence of the theoretical relationship of quantum, EDL, and total capacitances in eqn (1) is that $C_{\text {total }}<\min \left(C_{\mathrm{Q}}, C_{\mathrm{EDL}}\right)$, suggesting that in cases where the quantum capacitance is less than the EDL capacitance, the total capacitance may be increased by increasing quantum capacitance. To alter the DOS and thus to change the quantum capacitance, the most common and widely used way is doping. Nitrogen-doped graphene has been studied for many years and shows a higher capacitance than pristine graphene and porous carbon. ${ }^{16-24}$ Ruoff et al. attributed this capacitance enhancement to the increase in quantum capacitance, ${ }^{16}$ but Choi et al. explained the capacitance increase by the higher binding energy between the pyridinic group and the cation. ${ }^{18}$ So this topic is still controversial due to the complex structure of $\mathrm{N}$-doped graphene and the experimental difficulty of the accurate measurement of the quantum capacitance.

In this report, we aim to elucidate the role of $\mathrm{N}$-doping in the graphene capacitance. To simplify the complex structure, we separately study the three most common configurations in $\mathrm{N}$-doped graphene: graphitic, pyridinic, and pyrrolic. In N-doped carbons, these three configurations can be distinguished by $\mathrm{X}$-ray photoelectron spectroscopy (XPS) of N 1s binding energy, ${ }^{25}$ as experimentally observed. ${ }^{26}$ Herein we focus on a single-layer graphene sheet to elucidate the influence of $\mathrm{N}$-doping on quantum capacitance and EDL capacitance. 


\section{Computational methods}

Quantum capacitance is calculated by the electronic density functional theory (DFT) using implicit solvation model through the JDFTx code. ${ }^{27,28} \mathrm{~N}$-doped graphene is modeled by a twodimensional sheet under periodic boundary conditions; the lateral supercell is built up using repeating unit cells of graphene with a lattice parameter of $2.46 \AA$, close to the experimental value of $2.461 \AA$ in pristine graphene. The thickness of the vacuum slab is $20 \AA$ above the graphene sheet along the $z$ direction and filled with an implicit electrolyte (that is, a dielectric continuum). The generalized gradient approximation in the form of PerdewBurke-Ernzerhof functional is applied to describe the exchangecorrelation effect. ${ }^{29}$ Ultra-soft pseudopotential is used to describe the interaction between nuclei and valence electrons. ${ }^{30}$ The optimization of the ionic position is accomplished by the BFGS algorithm at the fixed lattice constant with a cutoff energy of 20 hartree for the planewave bases, a $6 \times 6 \times 1 k$-point mesh to sample the Brillouin zone, and $10^{-6}$ hartree tolerance in total energy for convergence. For accurate calculation of the density of states $D(E)$ near the Fermi level, a cutoff energy of 40 hartree and a $48 \times 48 \times 1 k$-point mesh are used. From $D(E)$, we can readily calculate the quantum capacitance from

$$
C_{\mathrm{Q}}=\frac{\mathrm{d} Q}{\mathrm{~d} \varphi}=\frac{e^{2}}{4 k T} \int_{-\infty}^{+\infty} D(E) \operatorname{sech}^{2}\left[\frac{E+\varphi}{2 k T}\right] \mathrm{d} E,
$$

where $Q$ is the excessive charge on the electrode and $\varphi$ is external potential. See ref. 14 for details of computing $C_{\mathrm{Q}}$.

Classical molecular dynamics (CMD) simulations were performed to obtain the EDL capacitance based on the same nitrogen-doped configurations as those used in the calculation of the quantum capacitance. As illustrated in Fig. 1a, the simulated channel system consists of a slab of $1.0 \mathrm{M} \mathrm{NaCl}$ aqueous solution enclosed between two electrodes. The separation between the two electrodes was set to $5.0 \mathrm{~nm}$ to ensure the bulk-like behavior of the electrolyte in the channel center. The SPC/E model was used for the water molecules; ${ }^{31}$ the LennardJones (LJ) parameters for $\mathrm{Na}^{+}$and $\mathrm{Cl}^{-}$were taken from the work of Smith et al. ${ }^{32}$ All atoms in the electrode were described by a polymer consistent force field $(\mathrm{PCFF})^{33-34}$ and were fixed in space during the simulation. The electrode-electrolyte interaction included the Coulombic contribution (accounted for the electrostatic interaction between atomic charges) and the van der Waals (vdW) contribution (accounted for from the LJ potentials). The LJ parameters for the vdW interaction between an electrode atom (type $i$ with LJ parameters $\sigma_{i i}$ and $\varepsilon_{i i}$ ) and an electrolyte atom (type $j$ with LJ parameters $\sigma_{i j}$ and $\varepsilon_{j j}$ ) were obtained by using Lorentz-Berthelot rules: $\sigma_{i j}=\frac{1}{2}\left(\sigma_{i i}+\sigma_{j j}\right)$ and $\varepsilon_{i j}=\left(\varepsilon_{i i} \varepsilon_{j j}\right)^{1 / 2}$.

The CMD simulations were performed in the NVT ensemble using the MD package GROMACS. ${ }^{35}$ Since the system has a slab geometry, the slab-PME method $^{36}$ was used here to compute the electrostatic interaction. The dimension vertical to the electrode was set to be 5 times the electrode separation to guarantee that the accuracy of the electrostatic force calculation (a)

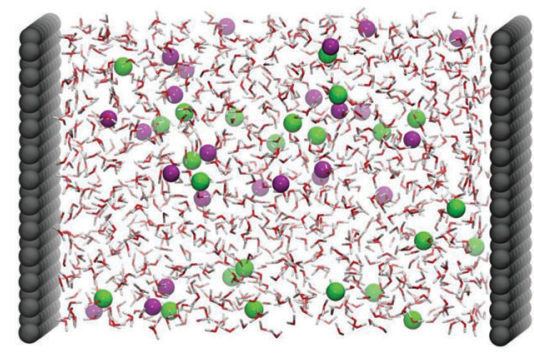

(b)
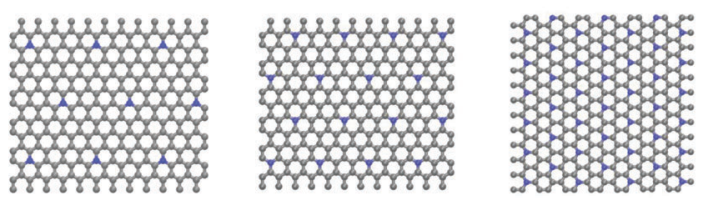

(c)
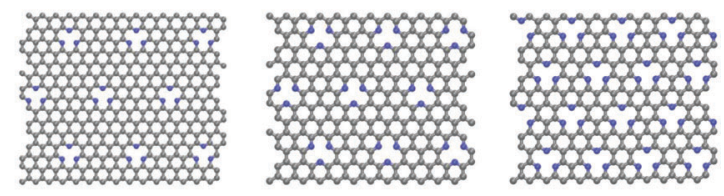

(d)
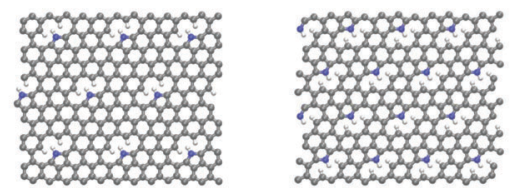

Fig. 1 (a) Snapshot of the MD simulation system; (b) graphitic N-doped graphene sheets with a doping percentage of $3.1 \%, 5.5 \%$ and $12.5 \%$ from left to right; (c) pyridinic $\mathrm{N}$-doped graphene with a doping percentage of $6.1 \%, 9.7 \%$ and $17.6 \%$ from left to right; (d) pyrrolic $\mathrm{N}$-doped graphene with a doping percentage of $3.2 \%$ and $5.9 \%$ from left to right. The concentration percentage is defined by the atom ratio from the number of atoms in the system ( $\mathrm{C}$ and $\mathrm{N}$ ). For instance, the $\mathrm{N}$ concentration is $\mathrm{N}$ atoms/( $\mathrm{N}$ atoms + $\mathrm{C}$ atoms). Grey, blue, purple, green, red and white balls denote the carbon, nitrogen, $\mathrm{Na}^{+}, \mathrm{Cl}^{-}$, oxygen and hydrogen atoms, respectively.

is comparable to that of the two-dimensional Ewald method. ${ }^{37}$ Each simulation was initiated at $800 \mathrm{~K}$ for $2 \mathrm{~ns}$, followed by $9 \mathrm{~ns}$ of equilibration at $298 \mathrm{~K}$, with temperature controlled by using a Berendsen thermostat. Another production run of 15 ns was performed for analysis. The time step of $2 \mathrm{fs}$ was applied and the atomic positions were saved every 4 ps. Previous reports ${ }^{38-39}$ showed that the 2 fs time step is sufficient enough to yield stable dynamics for the aqueous systems. Herein, we indeed found that the 2 fs time step afforded stable dynamics and stable electrostatic potential profiles in the water/graphene system. In addition, to ensure statistical accuracy, each simulation was repeated 3 times with different initial configurations (electrode configurations are shown in Fig. 1b-d).

Different electrical potentials were created by varying the surface charge densities of the electrode. The excess charges were evenly distributed to each atom. The final partial charge of each atom is composed of the excess charge and the partial charge from the force field. The MD calculation setup is similar to a previous work. ${ }^{40}$

We establish the total capacitance as a function of applied voltage $\left(\varphi_{\mathrm{a}}\right)$ by combining $C_{\mathrm{Q}}$ and $C_{\mathrm{EDL}}$ as described in 
the literature. ${ }^{14}$ First, we calculated the charging curves $(Q$ vs. $\varphi$ ) for both quantum and EDL capacitances separately: quantum capacitance as a function of $\varphi_{\mathrm{QC}}=\left(E-E_{\mathrm{Fermi}}\right) / e$ and EDL capacitance as a function of $\varphi_{\mathrm{EDL}}=\varphi-\varphi_{\mathrm{PZC}}$. Then, we used the interfacial charge $Q$ to connect the two capacitances by obtaining the corresponding potential drops $\varphi_{\mathrm{EDL}}$ and $\varphi_{\mathrm{QC}}$ from EDL and quantum capacitances, respectively, at the same surface charge. So the total applied potential drop $\varphi_{\mathrm{a}}=\varphi_{\mathrm{EDL}}+\varphi_{\mathrm{QC}}$ and the total capacitance is therefore $C_{\mathrm{tot}}=Q / \varphi_{\mathrm{a}}$. By varying $Q$, the charging curve for the total capacitance is obtained. ${ }^{41}$

\section{Results and discussion}

We first examine the quantum capacitance, then the EDL capacitance, and finally the total capacitance of $\mathrm{N}$-doped graphene electrodes.

\subsection{Quantum capacitance of different N-doping configurations at low concentration}

We calculated the quantum capacitance of the $\mathrm{N}$-doped graphene with different doping mole concentrations. The three most common configurations are chosen: graphitic, pyridinic and pyrrolic, ${ }^{26,42-44}$ as shown in Fig. 1b-d, respectively. The quantum capacitance and density of states (DOS) of these three N-doping configurations are plotted in Fig. 2 and 3, respectively. One can see that graphitic and pyridinic $\mathrm{N}$-doping configurations can greatly increase quantum capacitance, but pyrrolic $\mathrm{N}$ shows a "V"-shaped curve similar to pristine graphene.

The quantum capacitance shown in Fig. 2 can be explained by the $n$-doping and p-doping mechanisms in graphene sheets. ${ }^{45}$ In the pyrrolic $\mathrm{N}$-doping configuration, the $\mathrm{N}$ atom can donate an extra electron in the $\mathrm{P}_{z}$ orbital due to the formation of the $\mathrm{N}-\mathrm{H}$ bond but the associated $\mathrm{C}$ vacancy results in the loss of one electron in the delocalized $\pi$ bond. Thus, the total number of electron does not change compared to pristine graphene. This explains why the pyrrolic N-doping configuration shows a "V"-shaped quantum capacitance similar to pristine graphene; the DOS plots of pristine graphene and pyrrolic-N-doped graphene

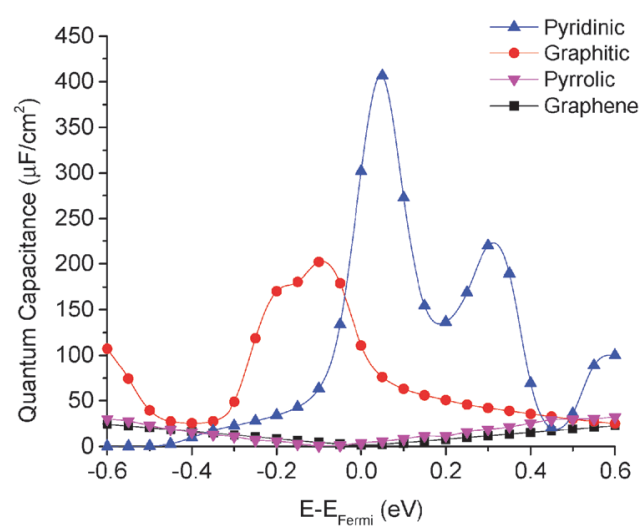

Fig. 2 Quantum capacitances of graphitic, pyridinic, and pyrrolic N-doped graphenes, with a mole fraction of nitrogen at $3.1 \%, 9.7 \%$, and $3.2 \%$, respectively, in comparison with that of pristine graphene.

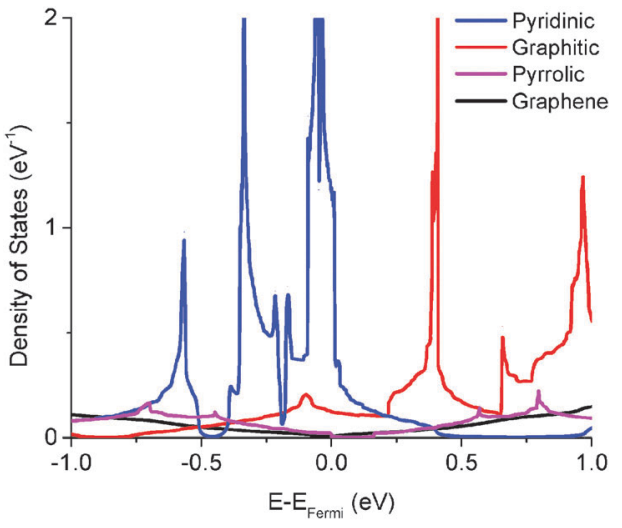

Fig. 3 Total density of states (DOS) of graphitic, pyridinic, and pyrrolic $\mathrm{N}$-doped graphenes, with a mole fraction of nitrogen at $3.1 \%, 9.7 \%$, and $3.2 \%$, respectively, in comparison with that of pristine graphene.

are also similar (Fig. 3). Graphitic N-doping can be regarded as an electron donator, which contributes one more electron to the delocalized $\pi$ bond than the $\mathrm{C}$ atom. Thus, the "Dirac Point" moves toward a higher energy position and the DOS near the Fermi level increases (Fig. 3), so the quantum capacitance increases. In pyridinic $\mathrm{N}$-doping, the number of electron on the $\mathrm{P}_{z}$ orbital of nitrogen does not change, but it causes a $\mathrm{C}$ vacancy and the system loses one electron compared to pristine graphene. Thus, the system is like a p-doping semiconductor, which shifts the "Dirac Point" down; the DOS near the Fermi level increases greatly (Fig. 3) and the quantum capacitance increases consequently.

\subsection{Quantum capacitance of graphitic N-doped graphene at different doping levels}

We computed the quantum capacitance of graphitic N-doping configuration with different doping concentrations and the results are plotted in Fig. 4. One can see that quantum capacitance exhibits considerable fluctuation, which is different from a smooth curve observed in a previous report. ${ }^{46}$ This may be due to our much denser $k$-point sampling in the Brillouin zone. We found that high doping concentration can increase quantum capacitance and

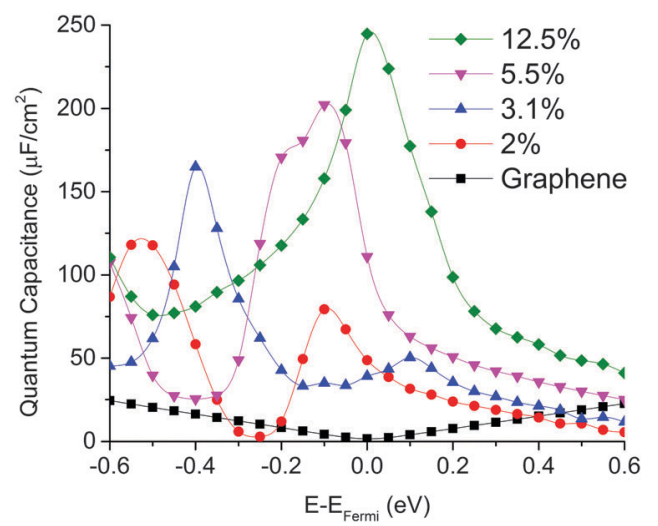

Fig. 4 Quantum capacitance of graphitic N-doped single layer graphene with different doping concentrations in comparison with that of pristine graphene. 
change the peak position, due to the corresponding change in the total DOS with concentration. As mentioned above, graphitic Ndoping can be treated as the n-doping type semiconductor, which will cause the "Dirac Point" move to the position below the Fermi level. Thus, as the concentration goes down, quantum capacitance decreases and the "Dirac Point" tends to move back to the Fermi level.

\subsection{Quantum capacitance of pyridinic N-doped graphene at different doping levels}

The quantum capacitance of pyridinic N-doped graphene with different doping concentrations is plotted in Fig. 5. In the pyridinic $\mathrm{N}$-doping configuration, the quantum capacitance enhancement is much stronger than the graphitic $\mathrm{N}$-doping under a comparable doping concentration. The quantum capacitance maximum can reach $400 \mu \mathrm{F} \mathrm{cm}{ }^{-2}$ in the pyridinic $\mathrm{N}$-doping configuration with the doping concentration of $9.7 \%$, but the quantum capacitance maximum is only $\sim 250 \mu \mathrm{F} \mathrm{cm} \mathrm{cm}^{-2}$ with $12.5 \%$ graphitic N-doping. However, the quantum capacitance fluctuation in the pyridinic configuration is much stronger. Unlike graphitic $\mathrm{N}$-doping, the quantum capacitance of the pyridinic $\mathrm{N}$-doping configuration will be very close to zero when the potential is below more negative than $-0.4 \mathrm{eV}$. This character in the pyridinic configuration does not depend on the doping concentration and will severely limit the total capacitance when the applied voltage is significantly negative. This extremely low quantum capacitance can be explained by the existence of a small gap at the "Dirac Point" caused by the p-type doping mechanism. One can see this gap in the DOS plot (Fig. 3) at $0.5-1.0 \mathrm{eV}$, corresponding to a negative electrode potential of about -0.5 to $-1.0 \mathrm{~V}$.

\subsection{The quantum capacitance of pyrrolic $\mathrm{N}$-doped graphene at different doping levels}

The quantum capacitance of pyrrolic N-doping graphene with different doping concentrations is plotted in Fig. 6. Unlike the graphitic and pyridinic N-doping configurations, the pyrrolic $\mathrm{N}$-doping configuration exhibits a "V"-shaped quantum capacitance similar to that of pristine graphene when the doping concentration

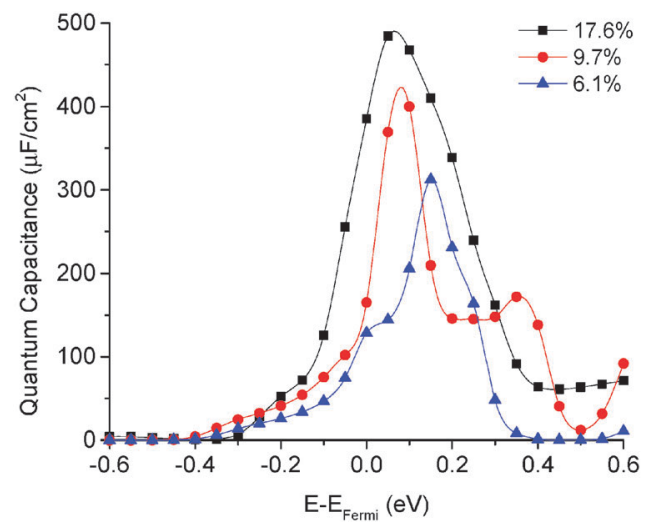

Fig. 5 Quantum capacitance of pyridinic N-doped graphene with different doping concentrations.

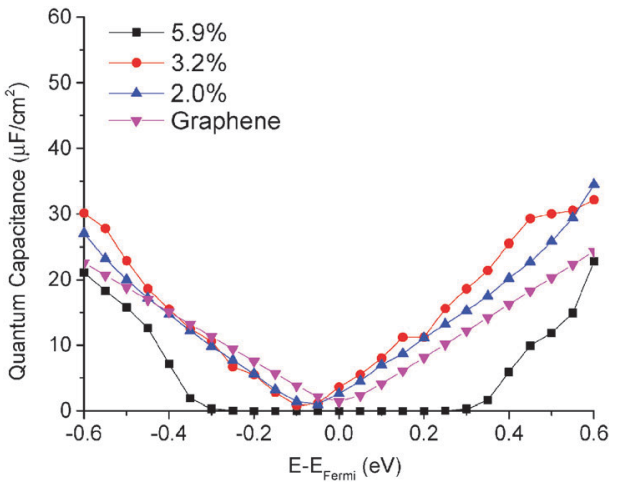

Fig. 6 Quantum capacitance of pyrrolic N-doped graphene with different doping concentrations in comparison with that of pristine graphene.

is low. As discussed previously, ${ }^{45}$ the pyrrolic $\mathrm{N}$-doping configuration has the same amount of electrons as pristine graphene and can be regarded as neither n-type nor p-type doped graphene due to the formation of the $\mathrm{N}-\mathrm{H}$ bond and vacancy. For a graphene or quasi-graphene type of 2D material, the total DOS always shows a "V" shape and the "Dirac Point" will be near the Fermi level. At low doping concentration, the correlation of pyrrolic $\mathrm{N}$-doped graphene and the vacancy between each unit cell can be treated as a small perturbation. Thus, the total DOS and quantum capacitance are very close to those of pristine graphene. However, things are different when doping concentration is quite large. In this situation (when the doping concentration is $5.9 \%$ ), quantum capacitance approaches zero near the Fermi level due to a gap in the total DOS as shown in Fig. 7.

\subsection{The EDL capacitance of $\mathrm{N}$-doped graphene in the $1 \mathrm{M}$ $\mathrm{NaCl}$ aqueous electrolyte}

In general, the EDL capacitance of carbon electrodes is $\sim 20 \mu \mathrm{F} \mathrm{cm}^{-2}$ in an aqueous electrolyte ${ }^{8}$ and $\sim 8 \mu \mathrm{F} \mathrm{cm}^{-2}$ in an ionic liquid. ${ }^{47-49}$ The voltage window is about $\mathrm{PZC} \pm 0.6 \mathrm{~V}$ in an aqueous electrolyte and about $\mathrm{PZC} \pm 1.5 \mathrm{~V}$ in an ionic liquid. Since the quantum capacitance that we explored lie mainly and is more reliable in $\mathrm{PZC} \pm 0.6 \mathrm{~V}$, we focus on the capacitance of doped graphene electrodes in an aqueous electrolyte.

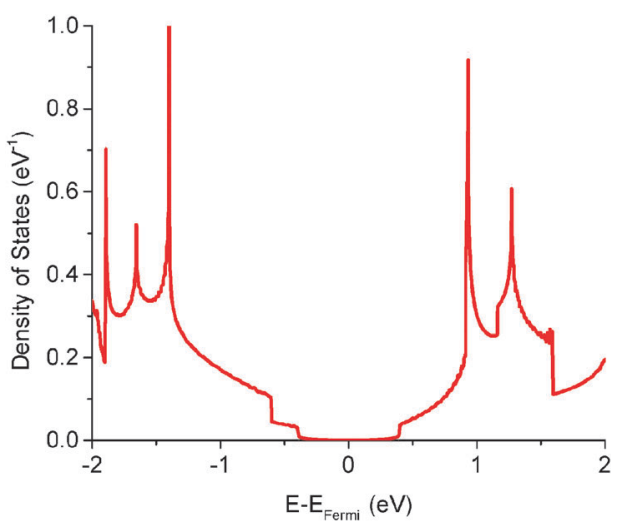

Fig. 7 Total electronic density of states of pyrrolic $\mathrm{N}$-doped graphene with the doping concentration of $5.9 \%$. 

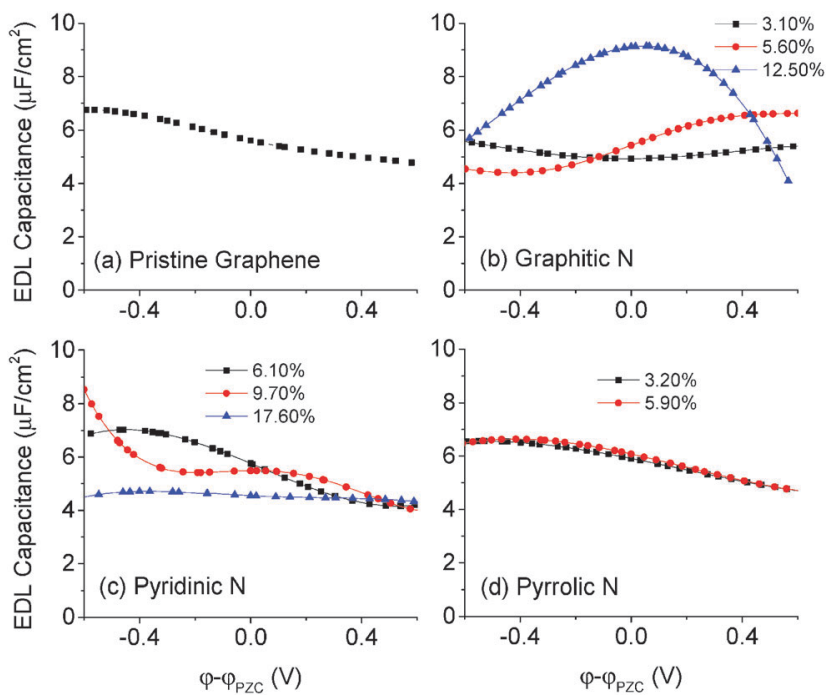

Fig. 8 Electric double-layer (EDL) capacitance of (a) pristine graphene, (b) graphitic, (c) pyridinic and (d) pyrrolic nitrogen-doped single layer graphene with different doping concentrations. The EDL capacitance is obtained from classical MD simulation for the $1 \mathrm{M} \mathrm{NaCl}$ aqueous electrolyte.

The differential capacitance of $\mathrm{N}$-doped graphene calculated by $\mathrm{MD}$ simulation in the $1 \mathrm{M} \mathrm{NaCl}$ aqueous electrolyte is plotted in Fig. 8. Comparing with pristine graphene, one can see that the EDL capacitance is almost the same for the pyrrolic configuration and perturbed to a small degree in the case of graphitic and pyridinic configurations. At high levels of doping, the differential capacitance is likely affected by the partial charge of dopants, causing the change in the EDL capacitance curves as shown in Fig. 8b and c. In most experimental studies, the total nitrogen content is lower than $10 \% ;^{42}$ in this case, Fig. 8 shows that the EDL capacitance does not change much in comparison with that of pristine graphene. This conclusion was also found previously for $\mathrm{N}$-doped graphene in an ionic liquid electrolyte. ${ }^{46}$

From the EDL capacitance shown in Fig. 8, we can conclude that the capacitance enhancement caused by the $\mathrm{N}$-doping effect is not due to the EDL capacitance. From eqn (1), we know that the total capacitance can be estimated from knowledge of both the quantum and EDL capacitances. Consequently, the quantum capacitance may be the key factor in explaining the capacitance enhancement of the N-doping effect. Specifically, the inequality $C_{\text {total }}<\min \left(C_{\mathrm{Q}}, C_{\mathrm{EDL}}\right)$ suggests that in those N-doped systems in which $C_{\mathrm{Q}}<C_{\mathrm{EDL}}$, the quantum capacitance will be the predominant determinant of the total capacitance. For systems in which $C_{\mathrm{Q}}>C_{\mathrm{EDL}}$, the EDL capacitance will be the predominant determinant of the total capacitance.

\subsection{Total integral and differential capacitance of $\mathrm{N}$-doped graphene in the $1 \mathrm{M} \mathrm{NaCl}$ electrolyte}

Now that we have computed both quantum and EDL capacitances separately, we can combine them to obtain the total capacitance. To compare with experiment more clearly, we used the charging curve based on $C_{\mathrm{Q}}$ and $C_{\mathrm{EDL}}$ to calculate the integral capacitance from $-0.6 \mathrm{~V}$ to $0.6 \mathrm{~V}$ (Table 1 ). One can
Table 1 Integral capacitance of different types of $\mathrm{N}$-doped graphene. The voltage window is from $-0.6 \mathrm{~V}$ to $0.6 \mathrm{~V}$

\begin{tabular}{ll}
\hline $\mathrm{n}$-Type & Integral capacitance $\left(\mu \mathrm{F} \mathrm{cm}^{-2}\right)$ \\
\hline Pristine graphene & 2.93 \\
Graphitic & 4.79 \\
Pyridinic & 5.34 \\
Pyrrolic & 3.05
\end{tabular}

see that, compared to pristine single-layer graphene, the integral capacitance increases by about $63 \%$ in graphitic and $82 \%$ in pyridinic $\mathrm{N}$-doping, but only by $4 \%$ in pyrrolic $\mathrm{N}$-doped graphene. The capacitance enhancement in our prediction is in good agreement with Fu et al.'s experimental work ${ }^{22}$ that showed capacitance enhancement of about 50\% for graphitic- and pyridinic-dominated configurations. Choi et al. applied nitrogen plasma to treat the graphene sample that may have caused more defects, and they found higher increases (over two times). ${ }^{18}$

We can obtain further insights from the total differential capacitance plotted in Fig. 9. Graphitic and pyridinic N-doped graphenes both have high and similar integral capacitance, but very different differential capacitance. Graphitic N-doped graphene exhibits a more stable and flat differential capacitance in the voltage window. Pyridinic N-doped graphene exhibits a larger differential capacitance than graphitic $\mathrm{N}$-doped graphene at a negative surface potential, but the differential capacitance goes down as the electrode potential goes up. In addition, the calculated total differential capacitance curves of graphitic and pyridinic are very close to the EDL capacitance obtained from by MD simulation. This is because for both graphitic and pyridinic nitrogens, quantum capacitances are much larger than EDL capacitances and the total charging dictated by the EDL charging. Unlike graphitic and pyridinic $\mathrm{N}$-doped graphenes, pyrrolic $\mathrm{N}$-doped graphene shows a "V"-shaped differential capacitance similar to that of pristine graphene and is in good agreement with the experiment. ${ }^{16}$

\subsection{Relevance to real N-doped carbon electrodes}

In real carbon or nitrogen-doped carbon materials, the electrode structure is very complicated. Experimentalists always measure

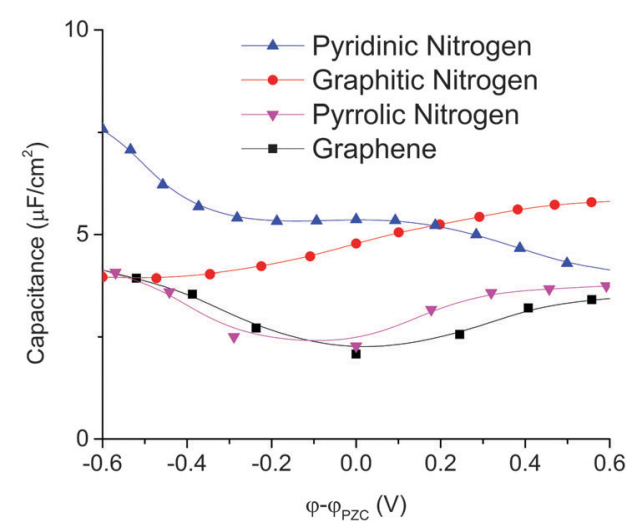

Fig. 9 Total differential capacitance of different types of $\mathrm{N}$-doped graphene for the $1 \mathrm{M} \mathrm{NaCl}$ aqueous electrolyte. 
BET surface area and pore size distribution to obtain some porosity information in carbon materials. For N-doped graphene, the commonly used way to identify different nitrogen types and their relative concentrations is XPS measurement of $\mathrm{N}$ 1s binding energy (B.E.). Our calculation has shown that different types of $\mathrm{N}$-doping configuration have different quantum capacitances and differential capacitances. In the real situation, experimentally obtained nitrogen doped graphene is a mixture of different $\mathrm{N}$-doping configurations. Thus, the integrated capacitance should depend on the relative proportion of each configuration, which can be well controlled by the synthesis method ${ }^{42-43}$ and characterized by XPS. It has been shown both experimentally and theoretically that pyridinic and pyrrolic nitrogens have stronger thermal stability than graphitic nitrogen in high temperature. ${ }^{18,22-23,50}$ Combining the thermal stability and our capacitance study, one can arrive at important implications for capacitance enhancement in $\mathrm{N}$-doped graphene systems. The most important implication is that one should increase the concentrations of pyridinic and graphitic nitrogens and decrease that of pyrrolic type to increase capacitance. Since graphitic nitrogen is much less stable than the other two types, one should focus on the pyridinic type if high-temperature treatment is needed in synthesis.

Our calculated quantum capacitance (Fig. 6) and differential capacitance (Fig. 9) of pyrrolic N-doped graphene both show a "V"-shaped capacitance vs. a voltage curve, which is very similar to that of pristine graphene. This calculation shows very good agreement with the experimental measurement of Ruoff et al. in the aqueous electrolyte; ${ }^{16}$ their XPS analysis showed that most nitrogen atoms are in the pyrrolic configuration. The large capacitance enhancement observed by $\mathrm{Choi}^{18}$ and Liu $^{17,19}$ is probably caused by graphitic and pyridinic $\mathrm{N}$-doping configurations.

\section{Summary and conclusions}

We have calculated the quantum, electric double-layer (EDL), and total capacitances of $\mathrm{N}$-doped graphenes of different $\mathrm{N}$ types and concentrations to investigate their effects on capacitances. The EDL capacitance was calculated by classical MD simulation and quantum capacitance by Kohn-Sham DFT. Graphitic and pyridinic configurations greatly increase quantum capacitance, which is proportional to the doping concentration. Pyrrolic configuration shows a " $V$ "-shaped quantum capacitance similar to that of pristine graphene, hence no enhancement. Classical MD simulations of the doped graphene electrodes in the $1 \mathrm{M} \mathrm{NaCl}$ aqueous electrolyte showed that $\mathrm{N}$-doping has relatively little effect on the EDL capacitance for the doping concentrations examined. The total capacitance, by combining quantum capacitance and EDL capacitance, exhibits good agreement with experimental results. When the pyrrolic configuration is dominant, the measured differential capacitance shows a "V"-shaped curve similar to pristine graphene. When the graphitic or pyridinic configuration is dominant, a large capacitance enhancement is observed.
In conclusion, graphitic and pyridinic nitrogens can greatly increase the total capacitance by increasing quantum capacitance and the pyrrolic configuration will limit the increase in the total capacitance. Given the much higher stability of pyridinic and pyrrolic nitrogens than graphitic nitrogen, one should dope more pyridinic nitrogen and avoid the pyrrolic type.

\section{Acknowledgements}

This research was sponsored by the Fluid Interface Reactions, Structures, and Transport (FIRST) Center, an Energy Frontier Research Center funded by the U.S. Department of Energy (DOE), Office of Science, Office of Basic Energy Sciences. Y. Z. gratefully acknowledges Guang Feng for help with the MD simulations. This research used resources of the National Energy Research Scientific Computing Center, a DOE Office of Science User Facility supported by the Office of Science of the U.S. Department of Energy under Contract No. DE-AC02-05CH11231.

\section{References}

1 L. L. Zhang and X. S. Zhao, Chem. Soc. Rev., 2009, 38, 2520.

2 Y. Wang, Z. Q. Shi, Y. Huang, Y. F. Ma, C. Y. Wang, M. M. Chen and Y. S. Chen, J. Phys. Chem. C, 2009, 113, 13103.

3 J. Chmiola, G. Yushin, Y. Gogotsi, C. Portet, P. Simon and P. L. Taberna, Science, 2006, 313, 1760.

4 A. B. Fuertes, F. Pico and J. M. Rojo, J. Power Sources, 2004, 133, 329.

5 C. Vix-Guterl, E. Frackowiak, K. Jurewicz, M. Friebe, J. Parmentier and F. Beguin, Carbon, 2005, 43, 1293.

6 M. D. Stoller, S. J. Park, Y. W. Zhu, J. H. An and R. S. Ruoff, Nano Lett., 2008, 8, 3498.

7 D. W. Wang, F. Li, Z. S. Wu, W. C. Ren and H. M. Cheng, Electrochem. Commun., 2009, 11, 1729.

8 M. D. Stoller, C. W. Magnuson, Y. W. Zhu, S. Murali, J. W. Suk, R. Piner and R. S. Ruoff, Energy Environ. Sci., 2011, 4, 4685.

9 V. Alzari, V. Sanna, S. Biccai, T. Caruso, A. Politano, N. Scaramuzza, M. Sechi, D. Nuvoli, R. Sanna and A. Mariani, Composites, Part B, 2014, 60, 29.

10 T. M. Kott, B. H. Hu, S. H. Brown and B. E. Kane, Phys. Rev. B: Condens. Matter Mater. Phys., 2014, 89, 041107.

11 P. Brooksby, A. Farquhar, H. Dykstra, M. Waterland and A. Downard, J. Phys. Chem. C, 2015, 119, 25778.

12 X. N. Shan, S. Chen, H. Wang, Z. X. Chen, Y. Guan, Y. X. Wang, S. P. Wang, H. Y. Chen and N. J. Tao, Adv. Mater., 2015, 27, 6213.

13 E. Paek, A. J. Pak and G. S. Hwang, J. Chem. Phys., 2015, 142, 024701.

14 E. Paek, A. J. Pak and G. S. Hwang, J. Electrochem. Soc., 2013, 160, A1.

15 J. Vatamanu, X. Ni, F. Liu and D. Bedrov, Nanotechnology, 2015, 26, 464001.

16 L. L. Zhang, X. Zhao, H. X. Ji, M. D. Stoller, L. F. Lai, S. Murali, S. Mcdonnell, B. Cleveger, R. M. Wallace and R. S. Ruoff, Energy Environ. Sci., 2012, 5, 9618. 
17 C. H. Zhang, L. Fu, N. Liu, M. H. Liu, Y. Y. Wang and Z. F. Liu, Adv. Mater., 2011, 23, 1020.

18 H. M. Jeong, J. W. Lee, W. H. Shin, Y. J. Choi, H. J. Shin, J. K. Kang and J. W. Choi, Nano Lett., 2011, 11, 2472.

19 H. L. Cao, X. F. Zhou, Z. H. Qin and Z. P. Liu, Carbon, 2013, 56, 218.

20 K. Wang, L. W. Li, T. Z. Zhang and Z. F. Liu, Energy, 2014, 70, 612.

21 Z. H. Wen, X. C. Wang, S. Mao, Z. Bo, H. Kim, S. M. Cui, G. H. Lu, X. L. Feng and J. H. Chen, Adv. Mater., 2012, 24, 5610.

22 B. J. Jiang, C. G. Tian, L. Wang, L. Sun, C. Chen, X. Z. Nong, Y. J. Qiao and H. G. Fu, Appl. Surf. Sci., 2012, 258, 3438.

23 L. Sun, L. Wang, C. G. Tian, T. X. Tan, Y. Xie, K. Y. Shi, M. T. Li and H. G. Fu, RSC Adv., 2012, 2, 4498.

24 Y. Q. Zou, I. A. Kinloch and R. A. W. Dryfe, J. Mater. Chem. A, 2014, 2, 19495.

25 W. J. Gammon, O. Kraft, A. C. Reilly and B. C. Holloway, Carbon, 2003, 41, 1917.

26 L. Y. Zhao, R. He, K. T. Rim, T. Schiros, K. S. Kim, H. Zhou, C. Gutierrez, S. P. Chockalingam, C. J. Arguello, L. Palova, D. Nordlund, M. S. Hybertsen, D. R. Reichman, T. F. Heinz, P. Kim, A. Pinczuk, G. W. Flynn and A. N. Pasupathy, Science, 2011, 333, 999.

27 S. A. Petrosyan, A. A. Rigos and T. A. Arias, J. Phys. Chem. B, 2005, 109, 15436.

28 K. Letchworth-Weaver and T. A. Arias, Phys. Rev. B: Condens. Matter Mater. Phys., 2012, 86, 075140.

29 J. P. Perdew, K. Burke and M. Ernzerhof, Phys. Rev. Lett., 1996, 77, 3865.

30 K. F. Garrity, J. W. Bennett, K. M. Rabe and D. Vanderbilt, Comput. Mater. Sci., 2014, 81, 446.

31 H. J. C. Berendsen, J. R. Grigera and T. P. Straatsma, J. Phys. Chem., 1987, 91, 6269.

32 D. E. Smith and L. X. Dang, Chem. Phys. Lett., 1994, 230, 209.

33 H. Sun, S. J. Mumby, J. R. Maple and A. T. Hagler, J. Am. Chem. Soc., 1994, 116, 2978.
34 H. Sun, Macromolecules, 1993, 26, 5924.

35 B. Hess, C. Kutzner, D. van der Spoel and E. Lindahl, J. Chem. Theory Comput., 2008, 4, 435.

36 I. C. Yeh and M. L. Berkowitz, J. Chem. Phys., 1999, $111,3155$.

37 T. Darden, D. York and L. Pedersen, J. Chem. Phys., 1993, 98, 10089.

38 A. A. Chialvo, L. Vlcek and P. T. Cummings, J. Phys. Chem. C, 2013, 117, 23875.

39 A. A. Chialvo, L. Vlcek and P. T. Cummings, J. Phys. Chem. C, 2014, 118, 19701.

40 G. Feng, R. Qiao, J. S. Huang, B. G. Sumpter and V. Meunier, J. Phys. Chem. C, 2010, 114, 18012.

41 C. Zhan, J. Neal, J. Wu and D. E. Jiang, J. Phys. Chem. C, 2015, 119, 22297.

42 H. B. Wang, T. Maiyalagan and X. Wang, ACS Catal., 2012, 2, 781.

43 Y. H. Lu, Y. Huang, M. J. Zhang and Y. S. Chen, J. Nanosci. Nanotechnol., 2014, 14, 1134.

44 R. Lv, Q. Li, A. R. Botello-Mendez, T. Hayashi, B. Wang, A. Berkdemir, Q. Z. Hao, A. L. Elias, R. Cruz-Silva, H. R. Gutierrez, Y. A. Kim, H. Muramatsu, J. Zhu, M. Endo, H. Terrones, J. C. Charlier, M. H. Pan and M. Terrones, Sci. Rep., 2012, 2, 586.

45 B. Wang, L. Tsetseris and S. T. Pantelides, J. Mater. Chem. A, 2013, 1, 14927.

46 E. Paek, A. J. Pak, K. E. Kweon and G. S. Hwang, J. Phys. Chem. C, 2013, 117, 5610.

47 J. Vatamanu, L. L. Cao, O. Borodin, D. Bedrov and G. D. Smith, J. Phys. Chem. Lett., 2011, 2, 2267.

48 J. Vatamanu, O. Borodin and G. D. Smith, J. Phys. Chem. B, 2011, 115, 3073.

49 J. Vatamanu, O. Borodin and G. D. Smith, J. Am. Chem. Soc., 2010, 132, 14825.

50 Z. Q. Tian, S. Dai and D. E. Jiang, Chem. Mater., 2015, 27, 5775 . 